\title{
A Framework for Predictive Modeling of Intra-operative Deformations: A Simulation-Based Study
}

\author{
Stelios K. Kyriacou ${ }^{1,2}$, Dinggang Shen $^{1}$, and Christos Davatzikos ${ }^{1,2}$ \\ 1 Department of Radiology, \\ 2 Center for Computer-Integrated Surgical Systems and Technology (ERC), \\ Department of Computer Science, \\ Johns Hopkins University \\ kyriacou@cbmv.jhu.edu, dgshen@cbmv.jhu.edu, hristos@rad.jhu.edu
}

\begin{abstract}
Deformations that occur between pre-operative scans and the intra-operative setup can render pre-operative plans inaccurate or even unusable. It is therefore important to predict such deformations and account for them in pre-operative planning. This paper examines two different, yet related methodologies for this task, both of which collect statistical information from a training set in order to construct a predictive model. The first one examines the modes of co-variation between shape and deformation, and is therefore purely shape-based. The second approach additionally incorporates knowledge about the biomechanical properties of anatomical structures in constructing a predictive model. The two methods are tested on simulated training sets. Preliminary results show average errors of $9 \%$ (both methods) for a simulated dataset that had a moderate statistical variation and $36 \%$ (first method) and $23 \%$ (second method) for a dataset with a large statistical variation. Use of the above methodologies will hopefully lead to better clinical outcome by improving pre-operative plans.
\end{abstract}

\section{Keywords: finite element modeling and simulation, registration tech- niques, deformable mapping}

\section{Introduction}

A fundamental problem encountered in several kinds of surgical procedures is that pre-operative plans cannot be accurately executed, due to deformations that occur between the pre-operative setup and the intra-operative environment. For example, in prostate therapy, patients are often imaged in the subpine position and operated on in the lithotomy position. Moreover, anatomical deformations can also be caused by the surgical instruments themselves. This problem is very significant in robotically assisted percutaneous therapy involving needle and catheter insertion, since a pre-operative plan based strictly on pre-operative image coordinates cannot be accurately executed by a robotic system, unless soft tissue deformation is predicted and/or tracked during the procedure. 
A number of investigators have used biomechanical models to predict intraoperative deformations in particular with respect to the brain shift during imageguided neurosurgery $[1,2,3]$. Nevertheless, there have been few attempts to use statistical training sets in addition to the biomechanical models (c.f. [1]). In this paper we present steps towards the development of a framework for predicting soft tissue deformation, assuming that the deformation of interest is observed in a training set, from which a predictive model is constructed. We investigate two different, yet related frameworks. The first one is referred to as shape-based estimation $(S B E)$ and it extends ideas that have been used in statistical shape models [4]. In particular, from the training set we find the principal modes of co-variation between shape and deformation, by applying a principal component analysis on vectors that hold jointly landmark coordinates and their respective deformed coordinates vectors. When presented with a new shape, which corresponds to the anatomy of the individual patient, we express it in terms of the principal eigenvectors via an optimization procedure. We thus simultaneously obtain the most likely deformation of the individual anatomy. Our goal here is to find the component of the deformation that can be predicted from the patient's shape, based on the premise that anatomy (e.g. bone, muscle, ligaments) to some extent determines or constrains possible deformations.

The second approach that we examine is referred to as force-based estimation $(F B E)$. It is based on the premise that often there is additional knowledge about the biomechanical properties of the deforming anatomy. Hence, this knowledge should be utilized. Accordingly, we find the modes of co-variation between shape and forces, rather than shape and deformation. (Forces are calculated from the observed deformation and elastic properties; the latter can be optimized for best prediction of deformation in the training set.) From the resulting forces, we then find the deformation via the biomechanical model, using finite elements.

At this stage of our work, we have not worked on a particular application, but rather we created simulated shapes and deformations, in order to test and compare our methodologies. We plan to use this method for prostate therapy.

\section{$2 \quad$ Methods}

In this section we first describe how we created a training set by sampling probability distributions for the shape, elastic properties, and force parameters, and feeding them to a finite element model. We then describe SBE and FBE. All of our experiments are on 2D shapes. However, the principles are applicable to 3D.

Creating a statistical sample. In order to develop and test our methodology, we created training sets using a biomechanical model. The training samples were created by loading a plain-strain $2 \mathrm{D}$ shape with a uniform pressure at the top and fixed boundary conditions at the bottom (see figure 1, left panel). The shape is comprised of five ellipse-like areas. Each ellipse represents a different anatomical region with possibly different elastic properties. The material behavior is assumed to be linear elastic with a Poisson ratio of 0.48 , which gives an almost incompressible behavior typical of soft tissues. The Young's moduli $E_{i}$ 
(where $i$ may refer to ellipse $i$ ) are within the range of the Young's moduli of very soft tissue (order of $10,000 \mathrm{~N} / \mathrm{m}^{2}$ ). Linear elasticity is not necessary but is just a convenient first approximation. ABAQUS/CAE [5] is used for the automatic creation of the geometry, the application of elastic properties and boundary conditions, meshing, and the solution of the resulting finite element model. Although linear materials were used, large deformation mechanics rendered the problem nonlinear. Gaussian distributions were sampled for all major parameters of the simulated training samples: pressure at the top, major and minor diameters for all ellipses, the Young's moduli, and positions of the 3 small ellipses. We created two sets of training samples, having relatively lower and relatively higher variation in parameters. The first training set included 20 samples and the second one included 40 samples.

Extracting Statistical Parameters. Both of the methods that we examine in this paper, namely SBE and FBE, use principal component analysis, although they apply it in a somewhat different way. Hence, we will describe the basic principle of both methods first.

Assume that a collection of points, perhaps landmarks, defining a shape are arranged in a vector $\mathbf{s}$, and another collection of vectors are arranged in the vector $\mathbf{q}$. The vector $\mathbf{q}$ represents displacements in SBE and forces in FBE. In the case of SBE the displacements correspond to all the points in $\mathbf{s}$, while in the case of FBE the forces correspond to points in $\mathbf{s}$ that are on the boundary of the shape. Consider the vector $\mathbf{x}$, created by concatenating $\mathbf{s}$ and $\mathbf{q}$. We apply principal component analysis in order to determine the modes of variation of $\mathbf{x}$ from the training set, in a way similar to [4]. This procedure is summarized next: - Align the training samples.

- Create the vector $\mathbf{x}_{i}$ for each sample $i$ out of $n$ samples.

- Calculate the mean shape vector $\mathbf{x}_{\text {mean }}$ and covariance matrix $\mathbf{C}$ and extract its eigenvalues matrix $\mathbf{D}_{\text {full }}$ (with the eigenvalues, $\lambda_{i}$, sorted by decreasing size) and eigenvectors matrix $\mathbf{V}_{\text {full }}$ (with eigenvectors $\mathbf{v}_{i}$ being the columns of the matrix).

- A new vector $\mathbf{x}$ can be created within this Statistical Shape Model:

$$
\mathbf{x}=\mathbf{x}_{\text {mean }}+\mathbf{V a}
$$

where $\mathbf{V}$ is the part of $\mathbf{V}_{\text {full }}$ that corresponds to $m$ largest eigenvalues and $\mathbf{a}$ is an $m$-dimensional coefficients vector (with elements $a_{i}$ ). In this work we use all the eigenvectors that have a non-zero eigenvalue.

Predicting q for a new shape. The procedure described above effectively determines the modes of covariation between the shape vector, $\mathbf{s}$, and the vector to be predicted, q. When presented with a patient's pre-operative images (target), we know the vector $\mathbf{s}_{t}$ ( $t$ stands for target), which represents the patient's undeformed anatomy. Our goal is then to predict the vector $\mathbf{q}$, which will determine the patient's deformed anatomy. However, the eigenvectors in $\mathbf{V}$ have dimensionality higher than that of $\mathbf{s}_{t}$, and therefore since we don't know $\mathbf{q}$, we cannot find $\mathbf{a}$ via projection on $\mathbf{v}_{i}$. Instead, we solve an optimization problem in which the vector $\mathbf{a}$ is found so that it yields a shape that best fits $\mathbf{s}_{t}$, while being 
most likely. The probability distribution of $\mathbf{a}$ is determined from the training set.

Specifically, for a given target shape, $\mathbf{s}_{t}$, we find the vector a that minimizes the following objective function:

$$
\mathcal{E}(\mathbf{a})=\left\|\mathbf{s}-\mathbf{s}_{t}\right\|^{2}+\mu\left(e^{0.5 \sum_{1}^{m} \frac{a_{i}^{2}}{\lambda_{i}}}\right)^{2},
$$

where $\mu$ is a relative weighting factor and

$$
\mathbf{s}=\mathcal{T}\left(\mathbf{x}_{\text {mean }}+\mathbf{V a}\right),
$$

with $\mathcal{T}(\cdot)$ representing the operation of truncating the second half of a vector (the components corresponding to $\mathbf{q}$ ). The first term in (2) seeks vectors that get as close as possible to the patient's undeformed anatomy. The second term is proportional to $1 / \operatorname{Prob}(\mathbf{a})$ and penalizes vectors a that are unlikely. The solution is found using the Levenberg-Marquardt [6] (nonlinear) optimization scheme. We start with a guess vector $\mathbf{x}_{\text {guess }}$ based on eq. 1 and $a_{i}=k \sqrt{\lambda_{i}}$, where $k$ is a constant e.g. 0.1. This procedure results in an estimate for the "missing part" of $\mathbf{x}$, namely $\mathbf{q}$.

SBE and FBE. The predicted vector $\mathbf{q}$ is different in SBE and FBE methods. In particular, in SBE, we set $\mathbf{q}=$ displacements, thus predicting deformation directly from the patient's undeformed anatomy. This is accomplished by defining point-correspondences in the undeformed and deformed configurations of the training samples.

In FBE, we set $\mathbf{q}=$ boundary forces. Since boundary force measurements are not directly available, this method is less straightforward and is described in more detail next. We start with some nominal elastic parameters, which in a real case scenario would be initially taken from the literature or perhaps from experimental data. From the displacement vectors that are available for each training sample and from the nominal elastic parameters, we calculate a forces vector $\mathbf{f}$ for each training sample, via a finite element solution. We then use these forces in place of the vector $\mathbf{q}$ and calculate the statistics of $\mathbf{x}$, as described previously. In principle, we can iterate this procedure, by varying the elastic parameters and each time evaluating the accuracy of the predictive model via jack-knifing, an approach which we plan to use in future work.

Having established the predictive model, when presented with a new target (i.e. the pre-operative anatomy of the patient), we use the predictive model to obtain force estimates. We do this by minimizing $\mathcal{E}(\mathbf{a})$, as described previously. We then feed these estimated forces into a finite element mesh created for that patient and, by using the nominal elastic parameters (or those determined from the training set, in future work) we calculate the displacement field, and therefore the deformed (intra-operative) anatomy. Note that if no additional constraints are imposed, the problem is ill-posed, in that there are infinite number of solutions differing by a rigid-body motion. Since rigid-body motion is not of concern in our application, and in order to make the solution unique, we applied 3 additional displacement constraints on arbitrarily selected points. 

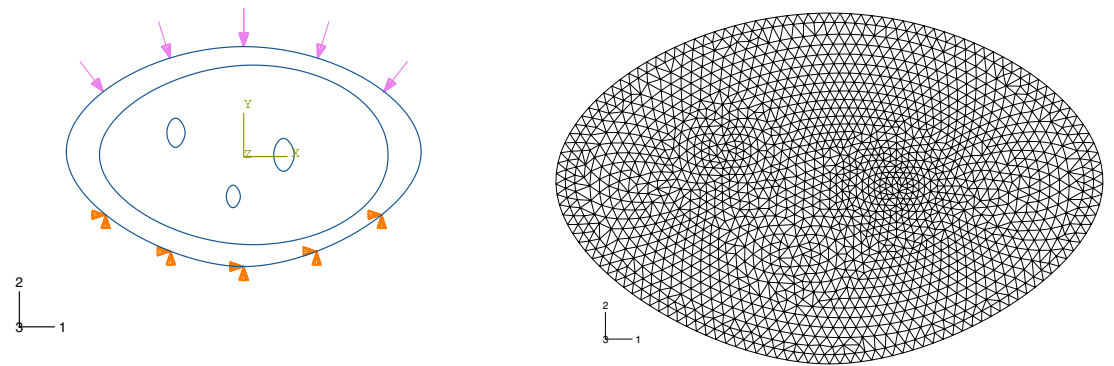

Fig. 1. Left: The model used in the phantom study. Uniform pressure is applied at the top and fixed boundary conditions are applied at the bottom of the large ellipse. Each ellipse may have its own elastic properties. Right: A sample finite element mesh with approximately 1500 nodes and 3000 linear triangular elements.

\section{Results}

Our first training set was comprised of 20 samples. The set was created by varying the Young moduli etc. according to Gaussian distributions, as explained in Methods. The standard deviation of each parameter was set equal to $1 / 20$ of the absolute of its mean. Four typical sample shapes from this sample set are shown in figure 2, top left, showing the extent of variation in the samples.

In order to test our predictive model, we used jack-knifing. In particular, we sequentially used 6 of the 20 training samples as test samples (targets), and for each target the remaining 19 samples were used to build the predictive model. The following estimates were used for the elastic properties for FBE: $E_{1}=E_{1 \text { mean }} * 2, E_{2}=E_{2 \text { mean }} * 0.5, E_{3}=E_{3 \text { mean }} * 2, E_{4}=E_{4 \text { mean }} * 0.5$. By $E_{1 \text { mean }}$ etc. we denote the mean values used in the Gaussian distribution to create the training dataset, and 1 denotes the 2 largest ellipse regions, while 2, 3 , and 4 denote the small lower right, lower left, and upper left ellipse regions respectively. By using material properties different than the true (mean) ones, we wanted to test the robustness of our predictive model to non-perfect estimates for the elastic parameters. Accordingly, we built 6 different predictive models, from which we calculated an average prediction error for each target. A typical example is shown in Fig. 2, which includes the results of both SBE and FBE. Note that each shape consists of 5 ellipses. The target undeformed shape is represented by a "." and the target deformed shape by an "o". The result of SBE is represented by an " $x$ " while the result of BFE by a "+". In this example, both methods achieved very good predictions, since the "+", "x", and "o" practically coincide. 
In order to examine the performance of these methods under higher variability in the sample set, we also created a second sample set with twice as high standard deviation. Fig. 3 shows a typical result from that sample set, with the same notation as in Fig. 2. Here, FBE outperformed SBE.

Tables 1 and 2 summarize the results for the first and second training datasets respectively. The error measure was obtained by calculating the root mean square error throughout all nodes on the boundaries of the five ellipses. The errors were normalized against the root mean square of the displacement vector for the same nodes.

\begin{tabular}{|r|r|}
\hline Error in SBE & Error in FBE \\
\hline \hline 0.115256 & 0.054368 \\
0.047286 & 0.066952 \\
0.100564 & 0.153132 \\
0.065547 & 0.056310 \\
0.081303 & 0.070515 \\
0.116419 & 0.153702 \\
\hline
\end{tabular}

Table 1. Normalized errors obtained for various targets for the low variation training dataset. The mean for SBE was 0.0877 and the mean for FBE was 0.0925 .

\begin{tabular}{|r|r|}
\hline Error in SBE & Error in FBE \\
\hline \hline 0.304303 & 0.053248 \\
0.126730 & 0.073419 \\
0.346008 & 0.566100 \\
0.532299 & 0.387028 \\
0.512355 & 0.048511 \\
\hline
\end{tabular}

Table 2. Normalized errors for various targets for the high variation training dataset. The mean for SBE was 0.3643 and the mean for FBE was 0.2257.

In order to examine the error introduced in FBE by the inherently approximating nature of finite element modeling, we tested the behavior of the two methods using a statistical sample set composed of 20 identical samples. SBE gave an error of 0.0000 , as expected, while FBE gave an error of 0.0032 . We conclude that the approximating nature of FEM is not a serious source of error in the methodology.

Computational time requirements. The most CPU intensive operation was the solution of the finite element problems. Each solution needed an average of 2 minutes on a $225 \mathrm{MHz}$ Octane SGI with $128 \mathrm{MB}$ RAM. In contrast, a 

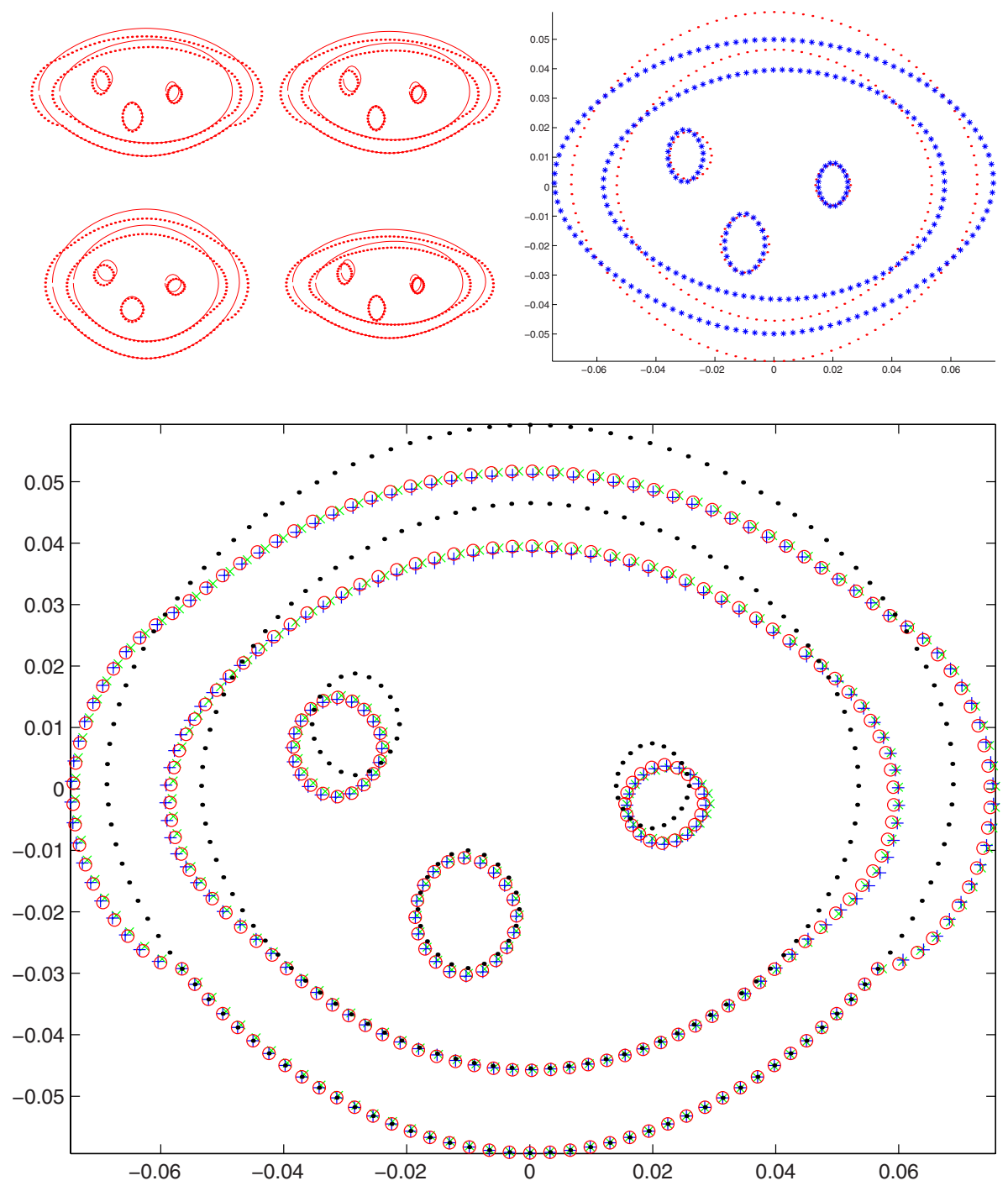

Fig. 2. Optimization results from a typical experiment in the low variation training dataset. Top Panel Left: Four sample shapes, showing the extent of variation in the samples. Solid and dotted lines depict the undeformed and deformed configuration respectively. Top Right: The initial undeformed shape used to initialize the Levenberg-Marquardt method is represented by "**", and this target's undeformed shape is represented by ".". Bottom Panel: The target deformed shape predicted by the two methods. SBE: "+". FBE: "x". True target deformed shape: "o". True target undeformed shape: ".". 
statistical solution was on the order of 10 seconds and an optimization solution was on the order of 60 seconds. Note that FBE required the solution of a finite element problem for each sample and the target so this method was slower than SBE by an order of magnitude.

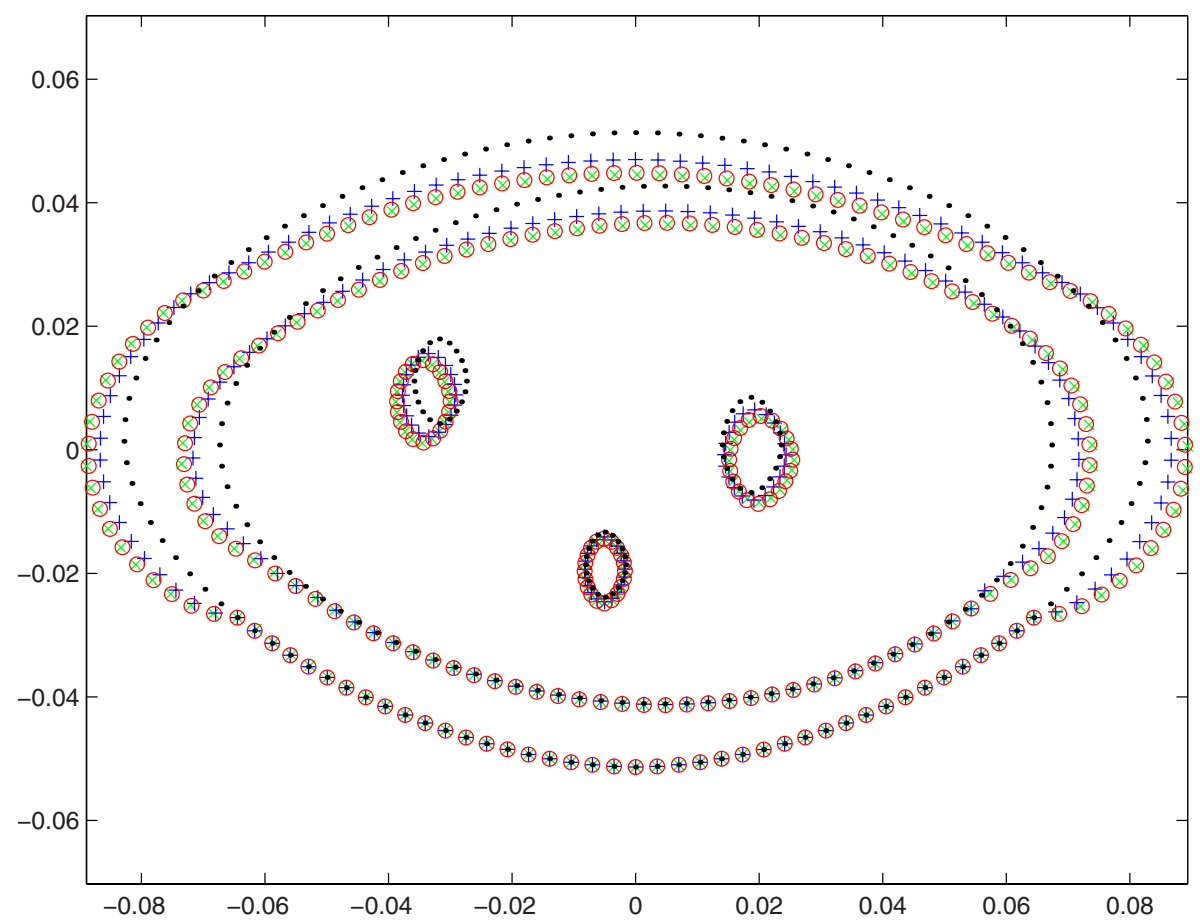

Fig. 3. Optimization results from a typical experiment in the high variation training dataset. SBE: "+". FBE: "x". Target deformed shape: "o". Target undeformed shape: ".".

\section{Discussion}

We presented a methodological framework for predicting deformations, in part using information obtained from a training set. We examined two methods. The first method (SBE) is purely shape-based and it constructs a predictive model based on the primary modes of covariation between anatomy and deformation. That is, it finds the component of deformation that can be predicted purely from knowledge of a patient's anatomy. The second method (FBE) utilizes knowledge about the biomechanical properties of the deforming anatomy, leaving the rest 
to a model analogous to the one used in SBE. Our preliminary results indicate that FBE outperforms SBE when large variations in anatomy, forces, and elastic parameters are present. However, both methods performed equally well under relatively lower variability. This is expected, since large deformation mechanics deviate from linearity, and therefore a linear statistical model should be expected to be relatively less accurate in that scenario.

FBE has the advantage that it utilizes knowledge about the biomechanical properties of anatomical structures. Moreover, it does not only estimate deformations on a discrete number of landmarks, but rather it determines a continuous displacement field. Its main drawback is the additional computational complexity, which in $3 \mathrm{D}$ is expected to be substantial.

Although it is clearly impossible to fully predict intra-operative deformations without additional intra-operative data, it is certainly beneficial to predict as much as possible of the deformation, and to account for this deformation during pre-operative planning. As pre-operative plans might need to be adjusted and recomputed intra-operatively, starting from a good initial guess should substantially reduce optimal planning computational time.

Future work will include the extension of our work to 3D. Potential applications include modeling of prostate and spine deformation, predicting deformations induced by needle insertion by using intra-operatively obtained forcefeedback measurements, and predicting prostate swelling in gene therapy.

\section{References}

[1] O.M. Skrinjar and J.S. Duncan. Real time 3D brain shift compensation. Proceedings of the 16th International Conference, IPMI'99 (Information Processing in Medical Imaging), Visegrad, Hungary, June/July 1999, Lecture Notes in Computer Science 1613, Springer. (Edited by A. Kuba and M. Samal and A. Todd-Pokropek), 1999.

[2] K.D. Paulsen, M.I. Miga, F.E. Kennedy, P.J. Hoopes, A. Hartov, and D.W. Roberts. A computational model for tracking subsurface tissue deformation during stereotactic neurosurgery. IEEE Transactions on Biomedical Engineering, 46(2):213-25, 1999.

[3] C.R. Maurer Jr., D.L.G. Hill, A.J. Martin, H. Liu, M. McCue, D. Rueckert, D. Lloret, W.A. Hall, R.E. Maxwell, D.J. Hawkes, and C.L. Truwit. Investigation of intraoperative brain deformation using a 1.5-T interventional MR system: preliminary results. IEEE Transactions on Medical Imaging, 17(5):817-25, 1998.

[4] T.F. Cootes, A. Hill, C.J. Taylor, and J. Haslam. Use of active shape models for locating structures in medical images. Image and Vision Computing, 12(6):355-65, 1994.

[5] Abaqus version 5.8. Hibbit, Karlsson, and Sorensen, Inc., USA, 1998.

[6] D.W. Marquardt. An algorithm for least-squares estimation of nonlinear parameters. J. Soc. Indust. Appl. Math., 11:431, 1963. 